\title{
Very Wideband, Compact Microstrip Bandstop Filter Covering S-Band to Ku-Band
}

\author{
Kamaljeet Singh and K. Nagachenchaiah \\ MEMS Development Divison, Semiconductor Laboratory (SCL), S.A.S Nagar, Chandigarh 160071, India \\ Correspondence should be addressed to Kamaljeet Singh, kamaljs@scl.gov.in
}

Received 2 September 2010; Accepted 22 November 2010

Academic Editor: Yong-Woong Jang

Copyright $\odot 2010$ K. Singh and K. Nagachenchaiah. This is an open access article distributed under the Creative Commons Attribution License, which permits unrestricted use, distribution, and reproduction in any medium, provided the original work is properly cited.

\begin{abstract}
This paper reports a wide bandwidth planar bandstop filter with improved RF characteristics. The proposed filter on alumina is realized incorporating tapped open stub along with spurline topology. Further, stepped impedance resonator (SIR) approach has been introduced in the tapped stubs to achieve wider band performance with improved selectivity. The proposed topology effectively controls the transmission poles. Fabrication of this BSF has been carried out on glass substrate showing minimal effect of permittivity variation on bandwidth performance. This validates the applied approach with achievable bandwidth of more than $100 \%$ ranging from S- to Ku-band. Close agreement with simulation and practical results have been demonstrated with measured insertion loss of less than $1 \mathrm{~dB}$ and attenuation loss better than $30 \mathrm{~dB}$ at C-band.
\end{abstract}

\section{Introduction}

Band stop filters find applications in oscillator and mixers to remove higher-order harmonics and other unwanted spurious signals. Duplexers and switches are comprised of BSF for filtering out unwanted signal along as they can interfere with the desired signals. Conventional methods to implement bandstop filters involve use of shunt stubs or stepped-impedance microstrip lines with large circuit size [1]. To reduce filter area, certain slow-wave structures, such as open-loop resonators, are widely adopted [2]. These traditional BSFs are normally having the narrow stop band response. As demand for wider stop band is gaining popularity alternative structures like photonic band gap (PBG) electronic bandgap (EBG), and the defected ground plane (DGS) are explored to cater the demand. Further to enhance the stop bandwidth, use of four or more cells of above-mentioned topologies is needed. However, this leads to a larger size and more transmission losses in the stop band. Alternatively, EBG and DGS require etching process on the backside ground plane in addition to position calibration using costlier lithographic techniques. Further its turnaround time is high and makes it incompatible to match with other topologies in the overall system configuration. Liu et al. [3] proposed spurline with cross-junction open stubs to have wider bandwidth with small size, but selectivity at one frequency end is compromised to cater for wider bandwidth. All the reported circuits are restricted to lower end of frequency where the effects of losses are not prominent, making it easier to analyze. Wider bandwidth topology at higher frequencies as reported by Hsieh and Wang [4] covers $2.3 \mathrm{GHz}$ to $9.5 \mathrm{GHz}$ range but has limited bandwidth. Wang and Her [5] reported spur line with SIR approach but concentrated mainly on selectivity aspect rather than bandwidth enhancement.

This paper demonstrates for the first time, a simple spurline topology with tapped stepped impedance resonators- (SIR-) based open stubs. These stubs can be construed as a dual-behavior resonator (DBR), controlling the selectivity with placement of the attenuation zeros at the desired frequencies. Various simulation studies are carried out to study the effect of substrate permittivity, uniform impedance resonator, and so forth, on the performance of the proposed filter. Presented approach is validated with practical data by realization of this filter on both alumina and on glass substrate. The losses achieved are less than $1 \mathrm{~dB}$ with 


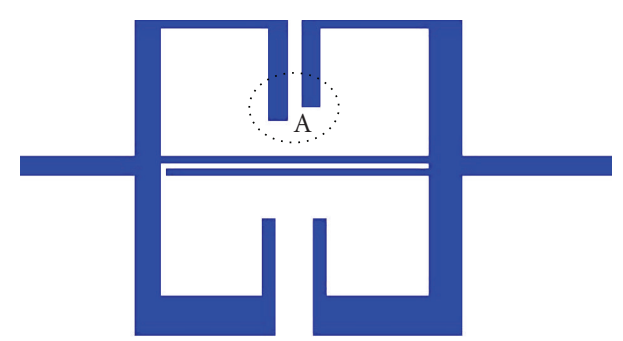

FIgURE 1: Proposed wide band BSF.

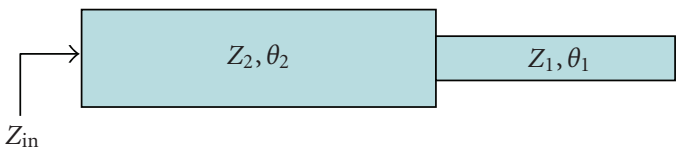

FIGURE 2: Basic structure of the open-ended SIR.

attenuation level of more than $30 \mathrm{~dB}$ on Alumina substrate. Design, simulation study, and fabrication aspects are detailed in this paper.

\section{Design Methodology}

A standard spurline filter design using theoritical equations and CAD tool has been carried out. The stubs are incorporated at the input and output ports for enhancing RF performance [6]. Impedances of the stubs are chosen so that overall ratio of impedances is not altered in tapped stubs. Effect of different impedance ratios is studied and bandwidth can be enhanced by varying thIS ratios. Symmetry of the structure is maintained and compactness is achieved by folding the stubs (Figure 1). Still, the selectivity improvement needs optimization which was achieved by using the coupled effects between the nonuniform lengths as shown in Figure 1 (marked A).

2.1. SIR Approach. The impedance of the SIR resonator shown in Figure 2 can be derived using the transmission line theory, as follows:

$$
Z_{\text {in }}=j Z_{2} \frac{Z_{2} \tan \theta_{2}-Z_{1} \cot \theta_{2}}{Z_{2}+Z_{1} \tan \theta_{2} \cot \theta_{1}}
$$

where $Z_{1}$ and $Z_{2}$ are the characteristics impedances of the two cascaded sections, $\theta_{1}$ and $\theta_{2}$ are the corresponding electrical lengths $\left(\theta_{1}+\theta_{2}=\pi\right)$. For determining the resonance frequency of the SIR, using ( 1 ) with $Z_{\text {in }}=0$ yields

$$
\tan \theta_{1}=R \cot \theta_{2},
$$

where $R$ is the impedance ratio of the SIR defined as

$$
R=\frac{Z_{1}}{Z_{2}}
$$

The resonance condition of the SIR can be adjusted by changing the width and length of $Z_{1}$ and $Z_{2}$. Frequency tuning is facilitated by adjusting $R$, as poles and zeros

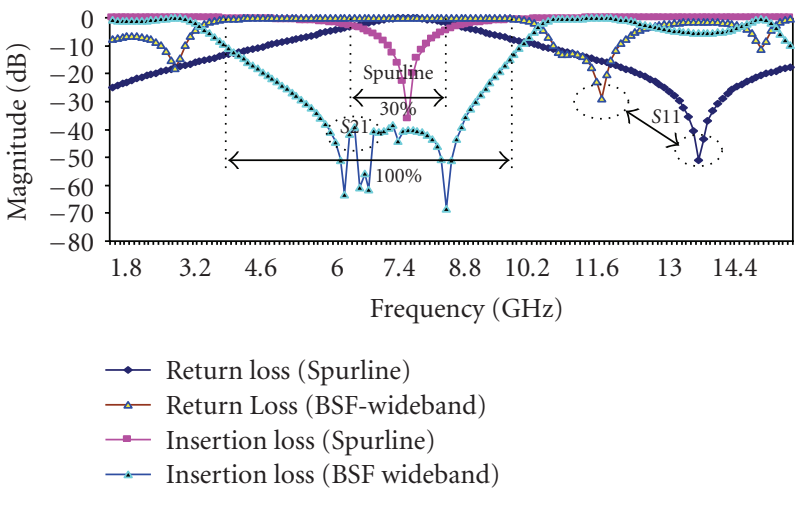

FIGURE 3: Comparison of the proposed topology with spurline.

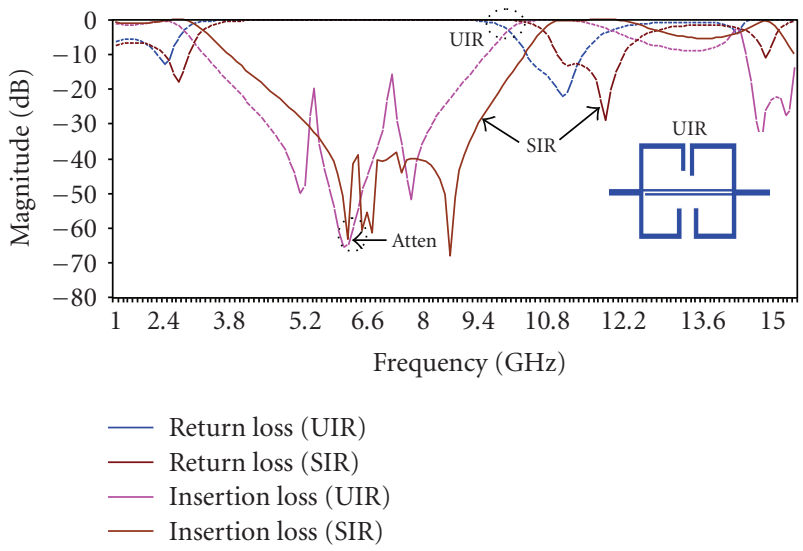

FIgURE 4: Comparison of the SIR stubs with UIR stub.

position directly depend on $R$. In the present design, three different impedances are simultaneously tuned to achieve wider band performance.

\section{Parametric Study}

Electromagnetic simulation study has been carried out by varying parameters like permittivity and impedances of the stubs [7]. All these studies have been carried out on 10 mils alumina substrate to cater high frequency of operation.

3.1. Bandwidth Comparison. Proposed BSF topology has been compared with standard spurline topology and it shows more than three times improvement in bandwidth. This is attributed due to the five transmission poles resulting in wider stop band attenuation as shown in Figure 3.

3.2. Effect of Uniform Impedance Resonator (UIR). Tapped open stubs based on SIR approach have been replaced with UIR stubs (inset Figure 4) as shown in Figure 4 keeping intact the overall dimensions and widths. UIR approach degrades the performance in terms of attenuation and selectivity as poles placement at the desired location cannot be controlled without optimization and keeping overall dimensions intact. 


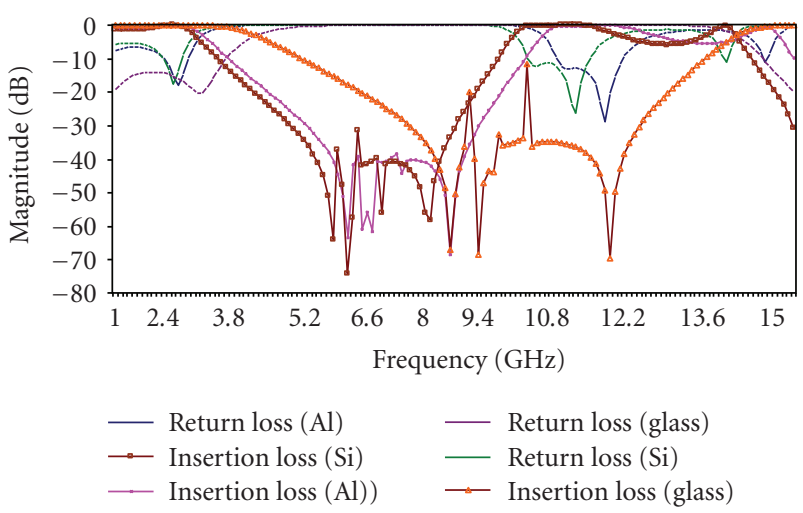

FIGURE 5: Effect of substrate permittivity on the RF characteristics of BSF.

3.3. Permittivity Variation. Due to inherent broadband nature of the proposed structure, variation of RF characteristics due to variable substrate permittivity has also been carried out. Structural dimensions optimized on alumina have been replicated on silicon and glass without dimensional changes and resulting EM simulation shows minimal influence of permittivity on the bandwidth.

As shown in Figure 5, Si $\left(\varepsilon_{r}=11.8\right)$ permittivity is close to Alumina $\left(\varepsilon_{r}=9.98\right)$, so performance is least affected and further drastically changing the permittivity to 4.82 (Glass), the shift in frequency is observed without affecting the inherent broadband characteristics.

\section{Filter Fabrication}

The band stop filter discussed is fabricated on the Pyrex 7740 glass substrate having thickness of $550 \mu$ and on 10 mil alumina substrate. The fabrication on alumina uses standard lithography processing steps but on glass substrate a extra step of thin film metallization is carried out. After subjecting to standard thin film substrate cleaning cycles, glass substrates are sputtered with thin layer of Cr (200$300 \AA$ ) followed by $7000 \AA$ of gold film on both sides of substrates. The sputtered metallization is electroplated with gold to the required thickness of $4.5 \mu \pm 3 \%$ and circuits are patterned using standard optical lithography and subtractive etching process.

The patterned substrate (both Al/Glass) is attached to gold metallized Kovar carrier plate using silver-based conductive epoxy. The carrier plate is mounted on test jig and RF connectors are connected by gold ribbon of 20 mils width and 1 mil thickness using parallel gap welding.

\section{Measurement Results}

Extensive CAD optimization has been carried out to achieve the desired specifications of broadbandwidth. Filters realized on the alumina and glass substrate are tested using the vector network analyzer PNA (8261A) from Agilent Technologies. Layout of the fabricated filter on alumina substrate is shown in Figure 6. The overall size comes around

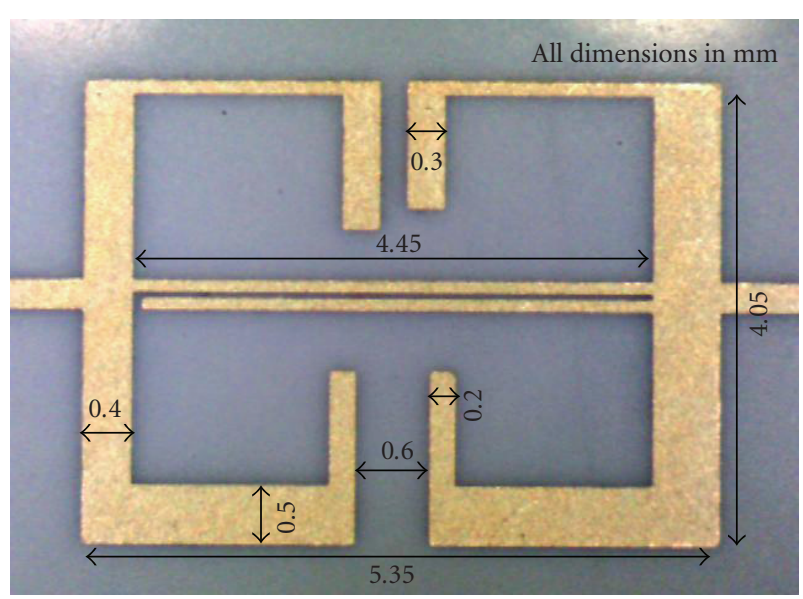

Figure 6: Layout of BSF on Alumina.

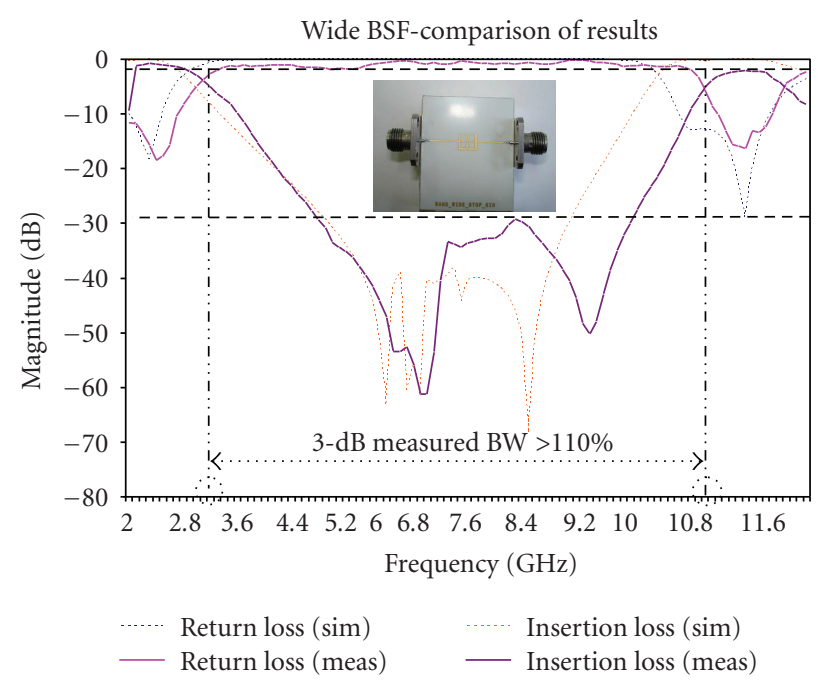

FIGURE 7: Simulated and measured results.

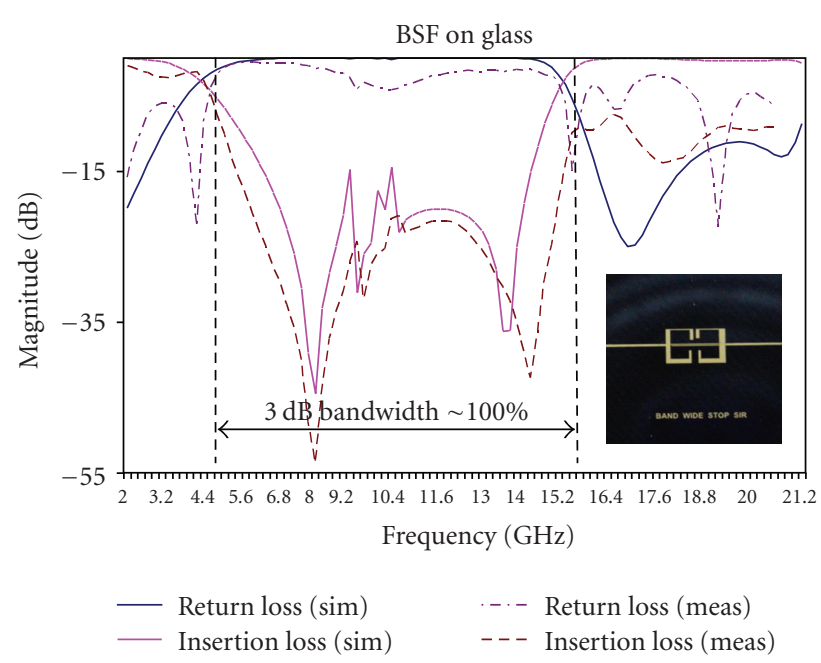

FIGURE 8: Measured versus simulated result on glass substrate. 
$7.5 \mathrm{~mm} \times 4.05 \mathrm{~mm}$ Comparison of simulated and measured results shows close agreement as shown in Figure 7.

Figure 8 depicts measured performance on the glass substrate patterned using same mask. It shows a very wide band performance indicating minimal effect of substrate permittivity on bandwidth.

Higher losses in the bandstop structure are associated with the increase of dielectric losses associated with glass $\tan \delta$ which needs parameters optimization.

\section{Conclusion}

Band stop filter with a wide bandwidth is proposed in this paper keeping intact the length as of standard spurline topology. The filter consists of one spurline and a pair of SIR stubs to achieve more than $100 \%$ bandwidth. Measured and simulated results are shown to be in close agreement. Proposed filter demonstrates better bandstop characteristics compared to existing reported structures retaining the compactness. Unique feature in the proposed topology is wide tolerance level for the substrate permittivity variation demonstrated by measurement and comparing its performances practically using both alumina and glass substrate. The proposed topology can also be easily implementable in MMIC.

\section{References}

[1] J. S. Hong and M. J. Lancaster, Microstrip Filters for RF/Microwave Applications, John Wiley \& Sons, New York, NY, USA, 2001.

[2] J. Shi, J. X. Chen, and Q. Xue, "Compact microstrip lowpass filter with wide stop-band integrating a bandstop structure in an open-loop resonator," Microwave and Optical Technology Letters, vol. 47, no. 6, pp. 582-584, 2005.

[3] H. W. Liu, Z. Shi, R. H. Knoechel, and K. F. Schuenemann, "Circuit modeling of spurline and its applications to microstrip bandstop filters," Microwave Journal, vol. 50, no. 11, pp. 126130, 2007.

[4] M. Y. Hsieh and S. M. Wang, "Compact and wideband microstrip bandstop filter," IEEE Microwave and Wireless Components Letters, vol. 15, no. 7, pp. 472-474, 2005.

[5] Y. Z. Wang and M. L. Her, "Compact microstrip bandstop filters using stepped-impedance resonator (SIR) and spurline sections," IEE Proceedings: Microwaves, Antennas and Propagation, vol. 153, no. 5, pp. 435-440, 2006.

[6] E. Rius, C. Quendo, C. Person, A. Carlier, J. Cayrou, and J. L. Cazaux, "High rejection C-band planar band-pass filter for a spatial application," in Proceedings of the 33rd European Microwave Conference, pp. 1055-1058, Paris, France, October 2005.

[7] "AC microwave, Linmic 6.2 +/N user manual". 

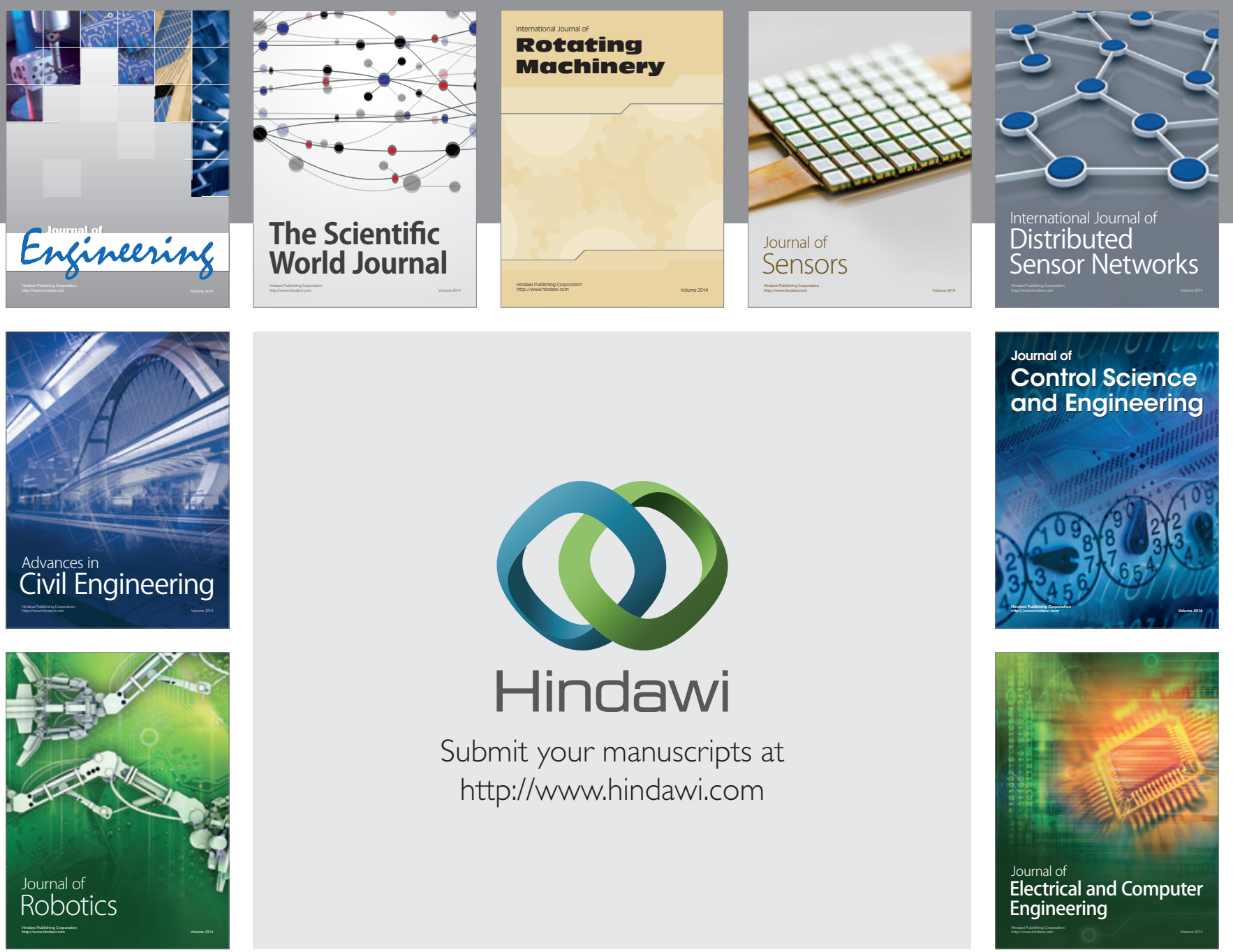

Submit your manuscripts at

http://www.hindawi.com
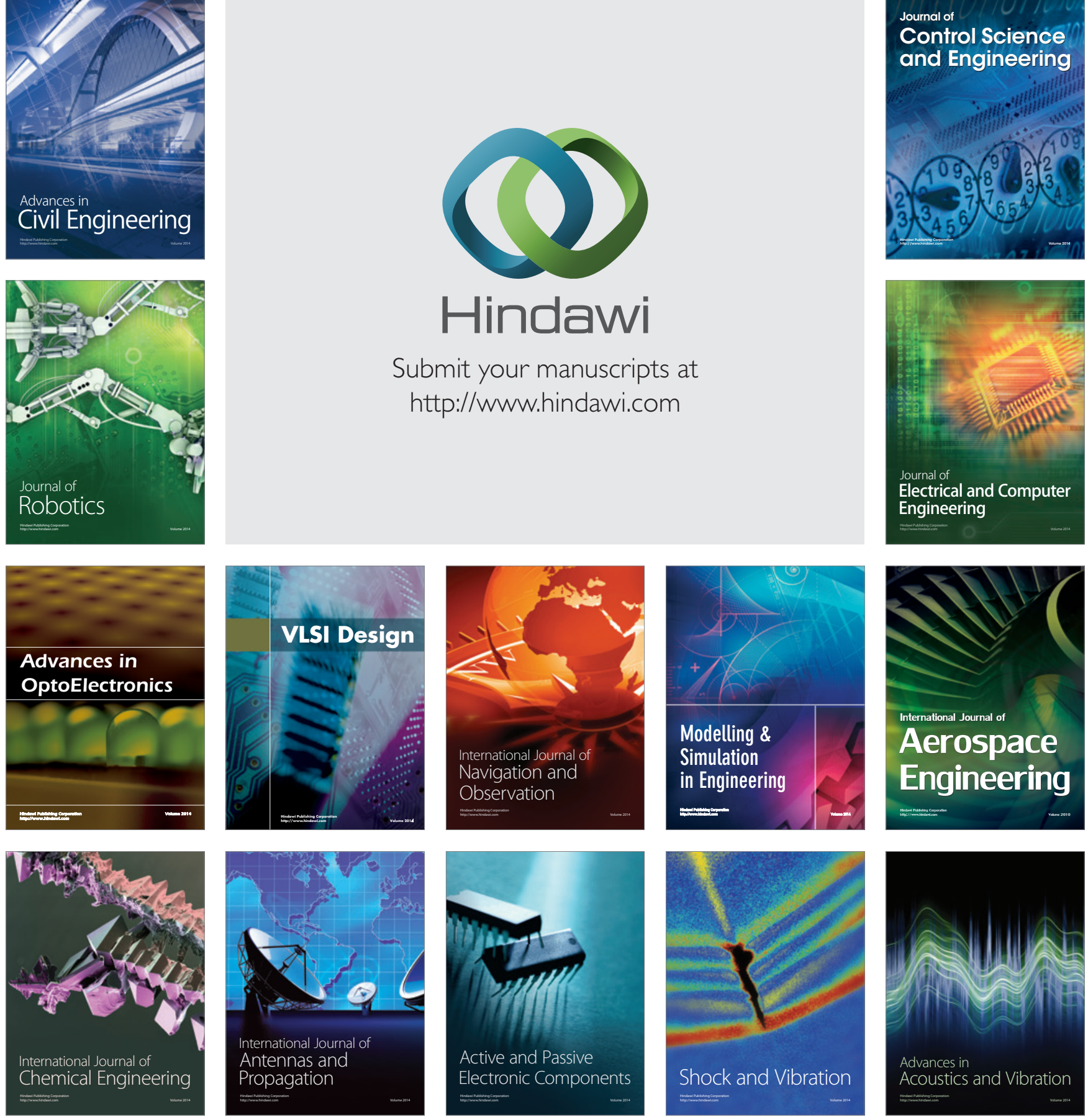